\title{
ARCHITECTURES AND GPS/INS INTEGRATION: IMPACT ON MISSION ACCOMPLISHMENT
}

\author{
Zdzislaw H. "Stan" Lewantowicz, Colonel, USAF \\ Deputy Director, Avionics \\ Wright Laboratory \\ Wright-Patterson AFB, OH 45433-6543
}

\begin{abstract}
The GPS contribution to navigational accuracy has been clearly established in military weapon systems. However the GPS signal provides enormously broader potential mission capability which has not yet been exploited. The GPS signal contains information, which, when properly combined with information from INS and other sensors, provides exceptionally high accuracy position, velocity, attitude, and time. These ten elements of information (three each in position, velocity, and attitude, and one in time) are common, in various combinations, to most of the avionics functions. When viewed from a system perspective, this high precision information, can be thought of as the integration basis, or a reference set, which offers opportunities for reconfiguration of the offensive, defensive, communication, navigation, and other sensors. Various integration architectures for fusion of these sensors can inherently enhance, enable, or severely limit these potential mission capabilities. The choice of integration architecture, can directly and profoundly affect performance, cost of integration, cost of ownership, and exploitation of much greater mission capability. This is illustrated with the GPS/INS integration example.
\end{abstract}

\section{INTRODUCTION}

The GPS potential to contribute to the military mission is no longer in question. The acceptance by the USAF Major Commands was already well established approaching the end of 1990 . Former commander of the Tactical Air Command (TAC) General Russ stated during the "TAC Day" in July 1990 (in essence): "GPS is here to stay, we have tried it, our pilots love it, get it in our aircraft as quickly as possible." The Operation Desert Storm simply validated and amplified this acceptance. Numerous articles in the civil, technical, and military journals have expounded on the GPS contribution to that operation. One defense journal, Defense News, quoted senior Defense officials that of the numerous success stories of the various space system asset contributions to that desert war, that of GPS was most significant. In the
Global View section of the June 1991 edition of the GPS World, in an article titled "After the Storm: DoD Officials Back Selective Availability," USAF General Donald J. Kutyna, Commander in Chief of the U.S. Space Command, is quoted as saying: (it) "would be difficult to overstate the value of the Navstar GPS system during Desert Shield and Desert Storm operations" and "this system has proved to be an unqualified success."

A multitude and variety of commercial and civil GPS applications are exploding, which in turn are fueling the explosion of commercial GPS receiver hardware development. The interest in this tremendous capability is most clearly evident in the growth of attendance at the GPS/ION conferences and in the number and variety of commercial and civil GPS application products. Over the last four years the attendance has grown from about 400 to nearly 1400 . This growth is remarkable despite the fact that only slightly more than half of the space segment is operational and that a vigorous debate is ongoing in the civil sector over military control of GPS. Of significance to this discussion is the fact that the free enterprise spirit has produced a multitude of ingenious solutions to such a vast number and variety of commercial and civil problems.

Leveraging of GPS capability for military applications does not appear to be as vigorous as that for the commercial sector. In Operations Desert Shield and Desert Storm the Allied forces also demonstrated tremendous ingenuity in the use and application of the available GPS equipment. Although the GPS receiver equipment available to them performed very well, the system has the potential for providing significantly greater capability. Had the full GPS system potential been more flexibly exploited, the Desert Storm air campaign would have likely produced even more impressive results.

There are, to be sure, various factors affecting the difference in utilization of the GPS by the military services, especially when compared to that of the commercial and civil sectors. Some of these factors are

U.S. Government work not protected by U.S. copyright. 
clearly identifiable and discussed in this paper while others are not known or not clearly understood. The more demanding military operating environment and mission requirements are among the more obvious differences. However there are other factors, some of which are systemic. Of the systemic factors, perhaps the most significant one is that of avionics integration architectures. Although GPS/INS integration is used as an illustration of a systemic factor, the discussion is presented from a broader perspective of system level avionics integration. Avionics architecture options are examined from the standpoint of the cost of integration, cost of ownership, and the yet unexploited mission capability.

\section{FEDERATED ARCHITECTURES}

Historically, the aircraft avionics have evolved from dedicated, single function, mechanical sensor systems to, more recently, sensor systems which have become quite sophisticated in their functionality and accuracy. The earlier sensors have been developed, refined, and added to the aircraft as stand-alone (federated) devices which provided crew members the increased ability to perform their mission more effectively. The fusion of information from a multitude of these avionics subsystems is one of the required pilot (or crew member) skills. Instrument flying requires the pilot to methodically and rapidly "cross-check" several instruments that make up the "performance" and "control" instrument groups. He must absorb this multitude of information and make continuous and real-time decisions about the type and quantity of control inputs to apply in order to achieve the required flight path precision. In this case the pilot mentally performs the fusion of the various elements of information required for safe instrument flight. Incidentally the auto-pilot, when engaged, performs similar fusion and control decisions for a subset of the flying tasks.

Increases in the earlier avionics capabilities typically came from evolutionary increases in sensor accuracy, range, reliability, affordability, etc. The more recently developed sensors have imbedded in them quite impressive computer processing capabilities to enhance the sensor performance. In more recently developed sensors, functional outputs from other sensors are added to enhance their performance. However the provided information, being the output of another sensor, is typically processed rather than the unprocessed information available closer to the sensor detector. In the past there have been valid and practical reasons for this. Two of these reasons were the technology availability and standardization constraints.
In relatively recent past, computational and data bus technologies simply did not exist, or their performance was inadequate, or the mission did not require the added "complexity." With time, as technology advanced, the sensor performance increased, the computational and data bus capabilities were developed and increased, and the mission requirements "warranted" some communication of various outputs among the avionics black boxes. For example, INS velocity information was provided to the synthetic aperture radar (SAR) to enhance its motion compensation performance. These were the earlier stages of integration and could be called "limited integration" or "output integration." Numerous constraints limited the quality, quantity, type, and other attributes of information in that limited integration information exchange.

In addition to the various forms of computational and data bus constraints, affecting a particular avionics sensor subsystem, are the standardization constraints. For example, the USAF Standard INS has stringent specification for not only the required physical fit and function but also for the digital output format, rate, and content, including the data word length, precision, structures, time tagging, and other related parameters. It is a fact that some of these constraints are imposed by the current technology availability, such as the speed limitation (capacity), due to today's saturation, of the MILSTD 1553 data bus.

There are early examples of limited sensor integration, such as the central air data computer (CADC). The CADC provides altitude, airspeed, and rate-of-climb information. The integration exemplified by the CADC is in combining of the dynamic and static atmospheric pressure-sensor information and enhancing it with relatively sophisticated, but open loop, compensation algorithms to provide higher accuracy outputs than those possible from the basic sensors. However, in many of the other instruments the combining of information is nonexistent or limited. For example the GPS/INS integration, described in a later section, is one such example. Of significance here is the fact that each sensor evolved primarily from the necessity for standalone operation, that is without the necessity for communication of much information to or from other sensors. And where information was needed to be shared with other sensors, the format of that information was typically constrained to that available at the postprocessed output serving the primary sensor purpose.

Despite some challenges in providing for multiple requirements, this limited integration concept was successful in that performance of some sensors was improved, to a lesser or greater degree, by adding to it information from other sensors. Most of the information 
that was provided from other sensors was at the postprocessed output level or in the domain of output integration. This post-processing modifies the "raw" signal, available from the detector, in bandwidth, noise statistics, and other electronic compensation characteristics. Although theoretically it possible to "undo" the output processing if the original processing is known, this is not practical due signal-to-noise losses, distortions, and typically high cost. The impact of this post-processing is that it limits the degree to which the information from other sensors can be utilized, where appropriate, to improve the performance of other sensors. This federated architecture, with its limitations, is the prevalent environment that the military GPS user equipment is required to interface to.

\section{MILITARY GPS EQUIPMENT}

When the military user equipment segment of the GPS was fielded, the navigation function also was provided in a "black box" (really gray colored), Standard Rockwell Collins Receiver IIIA. This box was required to perform as a stand-alone instrument, while at the same time it output information for several other devices, such as the INS, the fire control computer, or fire control sensors. These outputs were defined under various standards, such as the MILSTD 1553, but other restrictions were also applied. For example, the GPS receiver did not include the pseudo-range and delta-range information on the MILSTD 1553 bus output. Security considerations prevent the output of the Selective Availability (SA) corrected precision pseudo- and deltarange information outside some boundaries of an avionics sensor container. Instead the Receiver IIIA outputs navigation position, velocity, and other data after significant processing by its own internal Kalman filter. This filter is based on a generic INS model that is not optimized to any particular INS type such as gimbaled or strapped-down system.

If an aircraft requires a navigation solution optimized to a particular INS or if other sensor information is required for an optimal navigation (or other variable) solution, this GPS receiver constraint limits the aircraft Kalman filter designer to a cascaded, and suboptimal, filter implementation. This results in significantly degraded performance and jam resistance, and requires a strongly disadvantageous GPS availability or visibility definition. Also use of GPS filtered position and velocity outputs to drive a separate aircraft Kalman filter (e.g. in a fire control computer) can lead to filter stability problems, since the GPS and the INS data are time correlated (Reference 1). This potential problem is minimized by typically processing GPS measurements in the aircraft filter at a rate much slower than they are output from the GPS receiver. This helps in reducing the correlation between GPS and INS measurements, but not without penalties.

A measurement update spacing of 10-12 seconds is sufficient when four satellites with good geometry are available. When fewer than four satellites are available, the GPS receiver filter outputs degrade and closely track the rapidly growing INS errors. To avoid even greater filter instability under these conditions the aircraft filter is designed to disregard inputs from the GPS receiver when fewer than four acceptable satellites are available.

Despite this limitation, it is important to emphasize that the addition of the GPS receiver to the air weapon systems increased the mission capability tremendously from that of INS alone, or non-GPS aided INS. The GPS Receiver IIIA was designed and installed in the aircraft (and other vehicles) essentially as a navigator. It provides an unprecedented improvement in navigation accuracy over that of the previously available from the INS, which was aided by barometric altimeters, doppler velocity sensors, and other navigation instrument outputs. Fundamentally this increase in navigation accuracy provided substantial increase in mission capability. Global scale navigation accuracies of $16 \mathrm{~m}$ position and $0.1 \mathrm{~m}$ velocity are a couple orders of magnitude better than possible before introduction of GPS. However this performance increase is limited when compared to the potential performance, robustness, jam-resistance, etc. of a properly integrated GPS/INS. Performance increase in the navigation function as well as other improvements and benefits remain largely unexploited.

\section{ADVANCED AVIONICS ARCHITECTURES}

In contrast to the federated avionics architectures, the integrated avionics architectures offer numerous advantages. These advantages can be understood from a broader perspective of avionics system flexibility, redundancy, fault tolerance, real-time reconfigurability, decreased life cycle cost, adaptability, and other attributes. These architectures consist of multi-function, software programmable modules in common avionics enclosures. Many of the mission functions can be performed by individual or groups of modules. This concept replaces the discrete functions of the federated avionics architectures. The Integrated Communications, Navigation, Identification Avionics (ICNIA) and the INtegrated Electronic Warfare System (INEWS), are examples of such architectures, and are the basis for the U.S. Air Force Advanced Tactical Fighter (ATF), recently designated as the F-22, and the U.S. Army's next generation helicopter the LHX. The U.S. Navy is considering this avionics architecture for its AX system. Selection of modular avionics standard is a topic of high interest to the North Atlantic Treaty Organization 
(NATO), in particular the Four Power Group consisting of United States, United Kingdom, France, and Germany. The essence of the question before this group is not whether integrated avionics should be considered, but what form should the standards take for the avionics architectures and for one of its key elements, the modules. Although there are several examples of integrated avionics, for the sake of illustration, the next discussion uses ICNIA as an example.

The essence of integrated avionics architecture consists of several groups of modules in a common enclosure. Some modules perform such basic housekeeping functions as power provision, integrity monitoring, and timing. Another set of modules may be dedicated to the radio frequency (RF) processing of the various waveforms and conversion into, or from, digital formats. These modules either inject into, or extract from the RF aperture the required information waveform to be transmitted or received. This function can be performed for any of the frequencies that fall in the designed RF spectrum and for a variety of waveforms on any of the RF modules. The digital processing module group consists of an assembly of high speed processors which are realtime reprogrammable for whatever processing requirements may exist at any particular time or phase of the weapon system mission. The DoD mandated Ada language is the basis for the software development and validation environment.

The essence of this architecture is that any of the dozen or so functions defined in the ICNIA suite can be performed by combinations of RF and processor modules at any point in time. The entire architecture is software controllable, thus reprogrammable as necessary. In fact if an additional requirement of a yet undefined RF waveform were identified, no additional hardware may be required. Simply a software module, which identifies the new waveform and makes provisions in the ICNIA architecture, provides the new capability. Only requirement is that a sufficient number of modules are provided to assure that the desired processing capacity and level of redundancy (fault tolerance) are available. It is important to reemphasize that ICNIA is only one example of the integrated avionics architectures, and that it is an example of the currently available technologies. There are other approaches and technologies at the Wright Laboratory's Avionics Directorate, and at other Government laboratories and agencies. Some technology efforts are extending the integrated avionics concept toward even stronger integration and reliance on commonality, multifunctionality of modules as well as apertures, computational resource sharing and distributed processing.
Many advantages result from the integrated architecture concept. The essentially raw signals are brought into a single framework where real-time management of the available resources can be exercised. This immediately offers opportunities for real time reconfiguration for a spectrum of requirements, be it to account for failures, or reconfigure to adapt to a changing mission phase, or to respond to changing mission requirement, such as just identified enemy threat requiring a different and immediate response.

Perhaps the most significant advantage of integrated avionics, as the main message of this paper, is that most of the signals are available in various forms and stages of processing within the physical confines of this architecture. The communication, navigation, and identification signals in ICNIA, the defensive avionics sensors signals in INEWS, and the offensive avionics sensors signals are available in a variety of forms, from the "raw" to the fully processed for a variety of integration approaches. Sensor fusion ranging from combining of two or three sensors to large scale fusion of a multitude of sensors is possible. In any of these cases, the availability of the "raw" (unprocessed) pseudo- and delta-range GPS measurements provides unprecedented opportunities. When the GPS is properly combined with INS, this two sensor subset produces a significantly more accurate, robust, jam-resistant, less costly navigation, attitude, and time reference set. This reference set is very important because not only does it provide superior navigation solution, but it also becomes an integration reference set, for combining (fusing) of information from other sensors.

When other sensor information, which contains either absolute or relative navigation, attitude, or time information, is processed jointly with that of GPS and INS, then additional benefits are available. For example the reference biases, and other errors, of these sensors are often observable and estimable, thus providing a crosscalibration function. For those sensors where this crosscalibration is significant, several opportunities immediately appear. For example, the absolute performance tolerances on those sensors perhaps could be relaxed, thus reducing the complexity, cost, and reliability of those sensors. In some cases more than one sensor performs a similar or an overlapping function, or measures information also measured by some other sensor. When the information from this ensemble of sensors is properly fused, perhaps some of those sensors are either redundant, or their omission could result in minor and acceptable mission performance degradation but at a significant reduction in cost. Another possibility may be the realization of a common and highly accurate time reference that could be derived from this joint fusion 
of signals which have the GPS time as a common reference. Also, when information from other sensors is jointly fused with that of GPS and INS, the reference set accuracy and robustness is further improved.

These are only few examples of what may be achievable by integrated architectures. It is essential to emphasize, that to realize this vision, system and component level modeling, analysis, and simulation are required to identify the system level sensitivities and perform various trade-offs.

The modularity of the integrated avionics architecture reduces the number of the required module types to be supplied by the logisticians and lends itself to a two-level maintenance process, compared to the threelevel maintenance needed for the current federated avionics architectures. This modularity lends itself well to a commonality across many weapon systems, thus further reducing the potential requirement for the multitude of black boxes uniquely designed for a particular mission and/or a weapon system. This commonality drives upward the numbers of modules to be produced, but of fewer types, thus reducing the production costs.

Incidentally, because a large segment of the capability required is in digital processing hardware, this modularity offers a strong opportunity for rapid upgrading of the computational capability to meet the ever increasing threat challenge. This could be thought of as the imbedded processor upgrading capability at its best, because the computational function is clearly and cleanly distinguishable from the rest of the system architecture. Thus this modular approach postures the military weapon systems for much more rapid absorption of the commercial computational capability explosion.

Recognizing that the current military weapon systems with federated avionics lack the ability to strongly leverage this commercial computational explosion is perhaps one of the greater frustrations felt by many of the developers, producers, and users of avionics. Recognizing this as a challenge some military services are examining the avionics imbedded computer standards and applications.

The embedded computers in most of current avionics systems account for the largest subset of the current avionics standards. Many of these standards cover the electrical and data formats representing the quantity, quality, and types of information made available within the sensors, as well as that presented at the sensor outputs. The current avionics standards are strongly constraining the upgrading and integration of the existing avionics functions. The key question that has been asked is: What role should the electronics industry standards have in the avionics imbedded computers? Although this question is far from being answered, the significant fact is that this question is formally on the table for debate and action. When the avionics standards for imbedded, and other, computational resources are more closely aligned with those of the industry, then the military systems can begin to more continually, and with less time lag, leverage the commercially driven computational explosion.

\section{GPS/INS INTEGRATION}

The GPS/INS integration is a special subset of the larger set of Advanced Avionics Architectures and sensor fusion discussion. It is treated separately because of the very synergistic properties that a properly integrated GPS and INS functions can provide. In many vehicles that do not, and may not have integrated avionics architectures, an opportunity exists for installing a properly integrated GPS/INS in a single box. The miniaturization of the GPS receiver processing is more than ready for imbedding into the INS enclosure. For those who may feel attachments to the GPS as the primary function, a possibility exists of imbedding the ring laser gyro (RLG) or perhaps soon the fiber optic gyro (FOG) INS into the GPS enclosure. The benefits derived from such an integrated system are increases in accuracy, jam resistance, reliability, fault tolerance, and decreases in cost of the required INS and certainly in life cycle cost. Additionally this approach avoids the security problem with the Selected Availability because the sensitive signal need not leave the box.

Generally speaking, for good sensor fusion unprocessed information produces better results, as discussed earlier. For an eloquent discussion of the synergistic benefits of proper integration of these two elegant and proven system, the GPS and INS, see the now classic, in this author's view, paper by Cox (Reference 2). The vehicle dynamic maneuvering and the potential jamming of the GPS signals require the proper integration of the GPS with an INS. The significantly more demanding performance requirements of the military mission environment drive the implementation solutions in somewhat different directions than those of the civil and commercial applications.

Several analyses and experiments support the discussion by Dr. Cox and have pointed out significant mission capability advantages of the proper integration. For example in the analysis in References 3 and 4 the integration is performed at the raw pseudo-range signal level. That analysis demonstrates that a significant level of navigational performance improvement can be realized for the case when fewer than four satellites are available 
and pseudo-range and delta-range measurements are processed in a single integrated aircraft Kalman filter. Incidentally, this same filter should also be used for ground alignment as well. In fact, this filter configuration continues to estimate all of the observable INS, GPS, and barometric altimeter measurement errors including the gyro and accelerometer misalignment errors throughout all flight phases from power-up.

That analysis demonstrates GPS/INS system operation with three satellites, to be nearly as good as with four satellites for many minutes after four satellites were available. Even with two or one satellites the system performance is surprisingly good, certainly much better than in the case where no outputs are provided from the Receiver IIIA when fewer than four satellites are available. Most interesting is the case where GPS measurements are only momentarily, but infrequently available. There the performance is only slightly degraded from that of a fully and continuously available GPS measurement set.

The quality of these error estimates improves significantly during any maneuver segments, be it horizontal or vertical. In fact the flight trajectory in that analysis is rather sterile in the sense that all maneuvers are perfect and the straight and level flight is precisely straight and level. In reality pilots, and to a lesser degree autopilots, are not physically capable to fly these segments as perfectly. Thus any rolling, pitching, yawing, or linear accelerations, even relatively small in magnitude should help the filter by making the less observable errors become more observable.

Additional significant performance improvement can be realized by incorporating as many significant error states into this single integrated Kalman filter as possible subject to the computational memory and thruput constraints. The performance of a 51-, 32-, 23-, and 18state filter is compared against the performance of a 98 state truth model baseline. The performance of 32- and 51 -state filters substantially outperforms that of the 23and the 18-state filters. The performance difference is accentuated when less than ideal GPS availability conditions exist, such as during periods of jamming. Significantly, the performance of any of the four filters, is substantially better than that of a cascaded filterdriving-filter federated architecture.

Thus a GPS/INS, properly integrated in a single box offers several advantages which are not possible with the federated GPS/INS integration. Those advantages range from significant reduction in life cycle cost to significantly better overall system performance. Placing the Kalman filter inside this box and on a "Kalman filter chip" should unconstrain this GPS/INS integration and unburden the fire control computer. The GPS jamming performance must be redefined, because even momentary lock-on to the GPS signals at relatively large (several minute) intervals provides performance of an essentially unjammed GPS! Of course the best solution is to integrate the GPS and INS with other avionics sensors in an integrated architecture.

\section{CONCLUSIONS}

The challenge for the military research and development community is to vigorously exploit the simultaneous arrival of the GPS, the explosion in computational capability, and availability of the integrated and modular avionics architectures for weapon systems. These factors offer unprecedented opportunities for much greater exploitation of avionics sensor fusion. With proper fusion of the multitude of information available from the variety of sensors aboard a weapon system, much greater benefits can be derived from the information contained in the GPS signal. This sensor fusion is a strong function of the avionics architecture and the variety of forms of information readily available to any of the fusion algorithms. The avionics architecture can enhance or degrade the information fusion outcomes. In integrated and well-fused sensor avionics architectures, in addition to significant increases in performance potential, the concepts of standardization, system failure, and mission capability require redefinition. Entire sensor suites can then be revisited with the question: are all of the current sensors required for military mission performance?

\section{REFERENCES}

1. Johnson, Gregory B. and Lewantowicz, Zdzislaw H., "Closed Loop Operation of GPS Aided INS," Proceedings, ION-GPS 90 Conference, Colorado Springs, CO, September 1990.

2. Cox, Duncan B., Jr., "Integration of GPS with Inertial Navigation Systems," Navigation, Journal of the Institute of Navigation, 1978.

3. Lewantowicz, Zdzislaw H., and Keen, Danny W., "GPS Aided Navigation Performance for Weapon Release With Fewer Than Four Satellites," Proceedings, Joint Services Data Exchange Conference, October 1990.

4. Lewantowicz, Zdzislaw H., and Keen, Danny W., "Graceful Degradation of GPS/INS Performance With Fewer than Four Satellites," Proceedings, Institute of Navigation, National Technical Meeting, January 1991. 